\title{
HYPERNOVA AND GAMMA-RAY BURST REMNANTS AS TEV UNIDENTIFIED SOURCES
}

\author{
Kunihito IOKA ${ }^{1}$, AND PETER MÉsZÁros ${ }^{2}$
}

\begin{abstract}
We investigate hypernova (hyper-energetic supernova) and gamma-ray burst (GRB) remnants in our Galaxy as $\mathrm{TeV}$ gamma-ray sources, particularly in the role of potential $\mathrm{TeV}$ unidentified sources, which have no clear counterpart at other wavelengths. We show that the observed bright sources in the TeV sky could be dominated by GRB/hypernova remnants, even though they are fewer than supernova remnants (SNRs). If this is the case, TeV SNRs are more extended (and more numerous) than deduced from current observations. In keeping with their role as cosmic ray accelerators, we discuss hadronic gamma-ray emission from $\pi^{0}$ decay, from $\beta$ decay followed by inverse Compton emission, and propose a third, novel process of TeV gamma-ray emission arising from the decay of accelerated radioactive isotopes such as ${ }^{56} \mathrm{Co}$ entrained by relativistic or semi-relativistic jets in GRBs/hypernovae. We discuss the relevant observational signatures which could discriminate between these three mechanisms.

Subject headings: cosmic rays — gamma rays: bursts — gamma rays: theory — radiation mechanism: nonthermal — supernova remnants
\end{abstract}

\section{INTRODUCTION}

Observations at a new wavelengths have always led in astronomy to new discoveries, such as radio pulsars, gammaray bursts (GRBs), etc. The $\mathrm{TeV}$ gamma-ray sky is likely to yield similar surprises, since among the rapidly growing number of $\mathrm{TeV}$ sources (Aharonian et al. 2006) the most abundant category is of a mysterious nature, the so-called $\mathrm{TeV}$ unidentified sources (TeV unIDs), which have no clear counterpart at other wavelengths (Aharonian et al. 2005, 2008; Mukherjee and Halpern 2005). Our knowledge of TeV unIDs is very limited:

(1) So far $N_{\text {unID }} \sim 10-30 \mathrm{TeV}$ unIDs have been observed.

(2) They generally lie close to the Galactic plane, suggesting a Galactic origin.

(3) They are extended, $\Delta \Omega \sim 0.05-0.3^{\circ}$.

(4) The flux is $\varepsilon_{\gamma} F_{\varepsilon_{\gamma}} \sim 10^{-12}-10^{-11} \mathrm{erg} \mathrm{s}^{-1} \mathrm{~cm}^{-2}$ at $\varepsilon_{\gamma} \sim 0.2$ $\mathrm{TeV}$.

(5) They have a power-law spectrum with index of 2.1-2.5.

(6) Some TeV unIDs have strong upper limits in X-rays with a TeV to X-ray flux ratio of $F_{\mathrm{TeV}} / F_{\mathrm{X}} \gtrsim 50$ from Suzaku (Matsumoto et al. 2007; Bamba et al. 2007) and in radio with $F_{\mathrm{TeV}} / F_{\text {radio }} \sim 10^{3}$ (Atoyan et al. 2006; Tian et al. 2008).

The high energy nature of $\mathrm{TeV}$ unIDs naturally leads us to consider the cosmic ray (CR) accelerators as their possible origin. Since the dominant CR sources are supernova remnants (SNRs), TeV unIDs may be related to SNRs, in particular old ones, which are expected to be less luminous at other wavelengths (Yamazaki et al. 2006). However, the required galactic energy budget of $\mathrm{TeV}$ unIDs is only

$$
4 \pi d^{2} \varepsilon_{\gamma} F_{\varepsilon_{\gamma}} N_{\text {unID }} \sim 10^{34-35}\left(\frac{d}{10 \mathrm{kpc}}\right)^{2} \mathrm{erg} \mathrm{s}^{-1},
$$

${ }^{1}$ KEK Theory Center and the Graduate University for Advanced Studies (Sokendai), 1-1 Oho, Tsukuba 305-0801, Japan

${ }^{2}$ Center for Particle Astrophysics, Dept. of Astronomy and Astrophysics, Dept. of Physics, Pennsylvania State University, University Park, PA 16802 which is much less than that of SNe,

$$
\frac{10^{50} \mathrm{erg}}{100 \mathrm{yr}} \sim 10^{41} \mathrm{erg} \mathrm{s}^{-1}
$$

implying a rarer type of source.

Long GRBs are actually rare $\mathrm{SNe}$ endowed with relativistic jets, which also are expected to leave SNR-like remnants (Perna et al. 2000; Ayal \& Piran 2001). Even so, the expected number of GRB remnants is so small that previous works have addressed only specific sources (Ioka et al. 2004; Atoyan et al. 2006). However, recent observations suggest that hyper-energetic SNe, the so-called hypernovae which are sometimes associated with GRBs such as SN1998bw/GRB980425, SN2003dh/GRB030329 and SN20031w/GRB031203 (Maeda and Nomoto 2002), occur more frequently than GRBs (Guetta \& Della Valle 2007). The hypernova rate may be even higher if we are missing GRB-unassociated hypernovae like SN1997ef. On the other hand, a larger fraction of SNe may be endowed with slower or semi-relativistic jets, causing only low-luminosity (LL) GRBs. Actually, a significant energy $\sim 10^{50} \mathrm{erg}$ was released in the form of a mildly relativistic ejecta in the very faint GRB980425/SN1998bw. The recently discovered LL GRB060218/SN2006aj could be also be produced by a slower or semi-relativistic jet (Toma et al. 2007; Waxman et al. 2007), occurring at a $\sim 10$ times higher rate than GRBs (Guetta \& Della Valle 2007; Liang et al. 2007; Soderberg et al. 2006c). Such semi-relativistic jets could be intermediate between choked and breaking-out jets emerging through the progenitor's outer envelope.

An interesting point in $\mathrm{TeV}$ unIDs is that their emission may be related with hadronic processes, since a simple leptonic process for $\mathrm{TeV}$ gamma-rays by inverse Compton (IC) would require too much synchrotron emission than what is observed in TeV unIDs. Therefore we consider the hadronic processes associated with the $\pi^{0}$ decay $(\$ 2.1)$ for the hypernova shocks, and with the $\beta$ decay mechanism ( $\$ 3.1$, as well as a new mechanism of accelerated radio-isotope (RI) decay ( $\$ 3.2$ ) for the GRB jets. In the RI decay model, TeV gammarays are produced by the Lorentz-boosting of the $\mathrm{MeV}$ decay gamma-rays of accelerated RI, such as ${ }^{56}$ Co entrained by the 
jets. To our knowledge, this is the first discussion of the decay of accelerated RI as a mechanism for generating TeV gammarays in astrophysical sources. In this case, the source of the gamma-ray energy is injected initially at the base of the jet. There is no need for target matter or photons, as in the usual processes such as the $\pi^{0}$ decay, inverse Compton (IC) or photodisintegration (Anchordoqui et al. 2007).

In this paper, we investigate the high energy implications of GRB/hypernova remnants for $\mathrm{TeV}$ sources, in particular for $\mathrm{TeV}$ unIDs. Even though the total number of GRB/hypernova remnants is less than that of SNRs, the observed number in the TeV sky could be larger $(\S[2.1)$. This in turn predicts more extended (and more numerous) TeV SNRs than currently observed, which may be discovered by expanding the search region even with the current instruments. We use the units $k_{B}=h=1$ and $Q_{x}=Q / 10^{x}$ in cgs units unless otherwise stated.

\section{HYPERNOVA SHOCKS}

\section{1. $\pi^{0}$ decay model}

The simplest and most plausible process for TeV gammaray emission associated with CRs is the $\pi^{0}$ decay from $p p$ interactions between the interstellar medium (ISM) and CRs accelerated by the SN/hypernova shocks. Although the $\pi^{0}$ decay for SNRs (Naito \& Takahara 1994; Drury et al. 1994) and GRB remnants (Atoyan et al. 2006) has been discussed in detail, there are few works dealing with $\pi^{0}$ decay in hypernova remnants, except for the specific source HESS J1303631 (Atoyan et al. 2006). Since hypernovae are more energetic than SNe and GRBs and more frequent than GRBs, it is worth considering their implications for TeV unIDs.

By scaling the SNR calculation (Naito \& Takahara 1994. Drury et al. 1994), we obtain a TeV gamma-ray flux

$$
\varepsilon_{\gamma} F_{\varepsilon_{\gamma}} \sim 10^{-12} \zeta_{-1} E_{51} n d_{10 \mathrm{kpc}}^{-2} \operatorname{erg~s}^{-1} \mathrm{~cm}^{-2},
$$

comparable to the observed values. Here $n$ is the ISM density in $\mathrm{cm}^{-3}, d_{10 \mathrm{kpc}}=d / 10 \mathrm{kpc}$ and $\zeta$ is the fraction of the total CR energy $E$ per logarithmic energy interval. Since the kinetic energy of hypernovae is huge, $E_{k} \sim 10^{52} \mathrm{erg}$, a CR energy of $E \sim 10^{51} \mathrm{erg}$ is reasonable, for a conventional CR acceleration efficiency of $\sim 10 \%$.

Since the flux in equation (3) is independent of time, most sources are likely old, e.g., $t_{\text {age }} \sim 10^{5}$ yr old. Such old SNRs are possible $\mathrm{TeV}$ unIDs because the maximum energy of primary electrons is so small that the leptonic emission is suppressed. As shown in Fig. 1, the synchrotron emission from secondary electrons can also be below upper limits for $\mathrm{TeV}$ unIDs in the radio to X-ray bands (Yamazaki et al. 2006; Atoyan et al. 2006).

Remarkably, the observed number of old hypernova remnants may be comparable to that of the more numerous old SNRs, if the observations are flux-limited. For old remnants, which are larger than the angular resolution [see equation (8)], the source size should be taken into account. As the search region is expanded, more background is included. The sensitivity is proportional to the inverse square of the background, for background-dominated counting statistics. Therefore, the flux sensitivity to an extended source with a physical radius $r$ is given by $F_{\varepsilon_{\gamma}}^{\text {extend }}=F_{\varepsilon_{\gamma}}^{\text {point }}\left(r / d \theta_{\text {cut }}\right)$, where $F_{\varepsilon_{\gamma}}^{\text {point }}$ is the sensitivity to a point source and $\theta_{\text {cut }}$ is the angular cut in the analysis (Konopelko et al. 2002; Lessard et al. 2001). With equation
(3) and

$$
F_{\varepsilon_{\gamma}}^{\text {point }}\left(\frac{r}{d \theta_{\text {cut }}}\right)<F_{\varepsilon_{\gamma}} \propto E n d^{-2}
$$

we have the maximum distance to a source as

$$
d_{\max } \propto E n r^{-1}
$$

and hence the observable volume in the Galactic disk is

$$
V \propto d_{\max }^{2} \propto E^{2} n^{2} r^{-2} \propto E^{8 / 5} n^{12 / 5},
$$

where we use $r \propto E^{1 / 5} n^{-1 / 5} t_{\text {age }}^{2 / 5}$, which is approximately correct even for the radiative phase of the remnants. Since the hypernova energy is $\sim 10$ times larger, the observable number of hypernova remnants is larger than that of SNRs by

$$
\frac{N_{\mathrm{HNR}}^{\mathrm{obs}}}{N_{\mathrm{SNR}}^{\mathrm{obs}}} \sim \frac{R_{\mathrm{HNR}} V_{\mathrm{HNR}}}{R_{\mathrm{SNR}} V_{\mathrm{SNR}}} \sim \frac{10^{-4} \mathrm{yr}^{-1} \cdot 10^{8 / 5}}{10^{-2} \mathrm{yr}^{-1} \cdot 1^{8 / 5}} \sim 0.4,
$$

where $R_{\mathrm{SNR}} \sim 10^{-2} \mathrm{yr}^{-1}$ and $R_{\mathrm{HNR}} \sim 10^{-4} \mathrm{yr}^{-1}(\sim 7 \%$ of the $\mathrm{SNe}$ Ibc rate) are the event rates of SNe and hypernovae, respectively (Guetta \& Della Valle 2007), and we assume a similar age $t_{\text {age }}$ and ISM density $n$ for both remnants. The actual numbers in our Galaxy would be $R_{\mathrm{SNR}} t_{\text {age }} \sim 10^{3}$ (SNRs) and $R_{\mathrm{HNR}} t_{\mathrm{age}} \sim 10$ (hypernova remnants) for $t_{\mathrm{age}} \sim 10^{5} \mathrm{yr}$. Since the observed number of TeV unIDs is also $\sim 10$, we may be reaching the farthest hypernova remnants in our Galaxy.

The angular size of the $t_{\text {age }} \sim 10^{5}$ yr old remnants,

$$
\frac{r}{d} \sim \frac{30 \mathrm{pc}}{d} \sim 0.2^{\circ}\left(\frac{d}{10 \mathrm{kpc}}\right)^{-1},
$$

is consistent with TeV unIDs for hypernovae, ${ }^{3}$ but the same may not be true for SNe since the observable distance $d_{\max }$ is smaller and hence the angular size is more extended $[r / d \sim$ $\left.2^{\circ}(1 \mathrm{kpc} / d)\right]$. Such an extended object can be found only if the search region is expanded to the degree scale in the analyses. Therefore, if TeV unIDs are hypernova remnants, we can predict more extended (and more numerous) TeV SNRs than observed, which may be discovered by expanding the search region even with the current instruments.

The flux of hypernova remnants could be further enhanced if the density $n$ is higher around hypernovae than around usual SNe. The GRBs and SNe Ic, which are the only type of core collapse SNe associated with GRBs and hypernovae, are far more concentrated in the very brightest regions of their host galaxies than are the ohter SNe (Kelly et al. 2007; Fruchter et al. 2006), suggesting that GRBs and hypernovae are associated with star forming regions with high density. Conversely, hypernovae could be runaway massive stars ejected several hundreds of parsec away from high density stellar clusters (Hammer et al. 2006). In this case, no flux enhancement is expected.

It is necessary to check any possible side-effects of this mechanism at other wavelengths which might violate the hypthesis that they remain unidentified. In particular we need to check what is the possible X-ray emission from electrons in these sources. We may constrain the electron acceleration in the old hypernova remnants. In Fig. 1, we have plotted the bremsstrahlung, synchrotron, and IC emission from primary electrons with the same spectral index $p=2.2$, for

3 The CR diffusion could increase the source size (Atovan et al. 2006), although the diffusion coefficient has large uncertainties. 


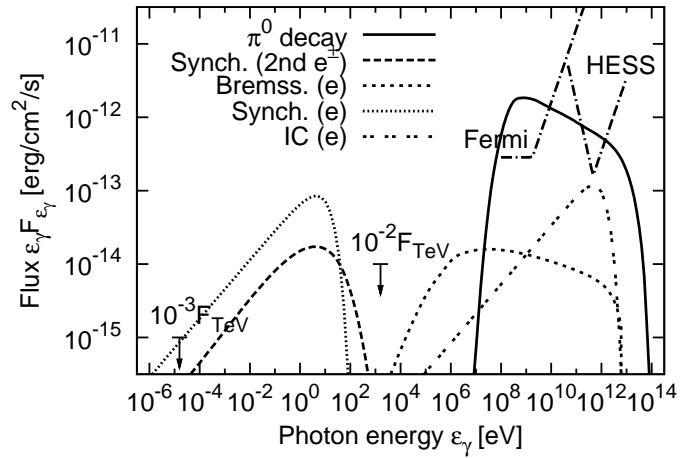

FIG. 1.- Flux from the $\pi^{0}$ decay via $p p$ interactions between the ISM and CRs accelerated by the hypernova shocks, compared with the Fermi and HESS sensitivities. We assume a remnant of age $t_{\text {age }}=10^{5} \mathrm{yr}$ at $d=10$ kpc with a CR energy $E=3 \times 10^{51} \mathrm{erg}$ and the CR spectral index $p=2.2$ in the energy range $m_{p} c^{2}<\varepsilon_{p}<10^{5} m_{p} c^{2}$. We also show the synchrotron emission from $\pi^{0}$ decay positrons and electrons for $B=3 \mu \mathrm{G}$, compared with the observational upper limits for an $\mathrm{X}$-ray to $\mathrm{TeV}$ flux ratio of $10^{-2}$ and a radio to TeV flux ratio of $10^{-3}$. We use a code of Kamae et al. (2006) for calculating $p p$ interactions. In addition, we also plot the bremsstrahlung, synchrotron, and IC emission from primary electrons with the spectral index $p=2.2$ and the electron-to-proton ratio of $(e / p)_{10 \mathrm{GeV}} \sim m_{e} / m_{p}$ at $10 \mathrm{GeV}$.

an electron-to-proton ratio of $(e / p)_{10 \mathrm{GeV}} \sim m_{e} / m_{p} \sim 10^{-3}$ at $10 \mathrm{GeV}$. The radio observations limit the $(e / p)_{10 \mathrm{GeV}}$ ratio to $\lesssim 10^{-3}$, which is somewhat smaller than the observations $\sim 0.02$. Note however that the injection of thermal electrons into the acceleration process is poorly understood, and the observed electron CRs might be produced by other sources such as usual SNRs or pulsars. In any case, the leptonic model for $\mathrm{TeV}$ unIDs seems unlikely. Note that we take the Coulomb losses for low energy electrons into account according to Uchiyama et al. (2002) (see also Baring et al. (1999)) and this makes the bremsstrahlung spectrum hard in the Xray band. On the other hand, the thermal bremsstrahlung emission is not bright in the X-ray band, in contrast with the usual young SNRs (Katz \& Waxman 2007), because the temperature of old SNRs is much below the X-ray energy, $T \sim 0.04 \mathrm{keV} E_{k, 52}^{2 / 5} n^{-2 / 5}\left(t / 10^{5} \mathrm{yr}\right)^{-6 / 5}$ (Reynolds 2008).

\section{GRB JETS}

\section{1. $\beta$ decay model}

A fraction of SNe/hypernovae is associated with long GRB and their jets. Another process for generating $\mathrm{TeV}$ gammarays is the (time-delayed) $\beta$ decay of the neutron component of the CR outflow accelerated by the jets followed by inverse Compton scattering (Ioka et al. 2004). We have applied this process to the SNR W49B (G43.3-0.2), which is a possible GRB remnant (Keohane et al. 2006; Miceli et al. 2006, 2008). Since the $\beta$ decay can occur outside the remnant, the jet-like emission would appear outside of the SNR. Note that the GRB-associated SNe are not necessarily energetic hypernovae since the luminosity distribution of GRBassociated $\mathrm{SNe}$ is statistically consistent with that of local SNe Ibc (Soderberg et al. 2006b).

As shown in Fig. 2] old jet remnants with $t_{\text {age }} \sim 10^{5} \mathrm{yr}$ are potentially $\mathrm{TeV}$ unIDs since the usual SNR emission goes down at this age, while electrons that emit $\sim \mathrm{TeV}$ gammarays (i.e., $\gamma_{e} \sim 10^{7}$ electrons) need $t_{\text {cool }} \sim 10^{5} \mathrm{yr}$ to cool (see Eq. (3) and (4) in Ioka et al. 2004). (However a spectral cutoff at $\sim 10 \mathrm{TeV}$ may be seen at this age.) Then the

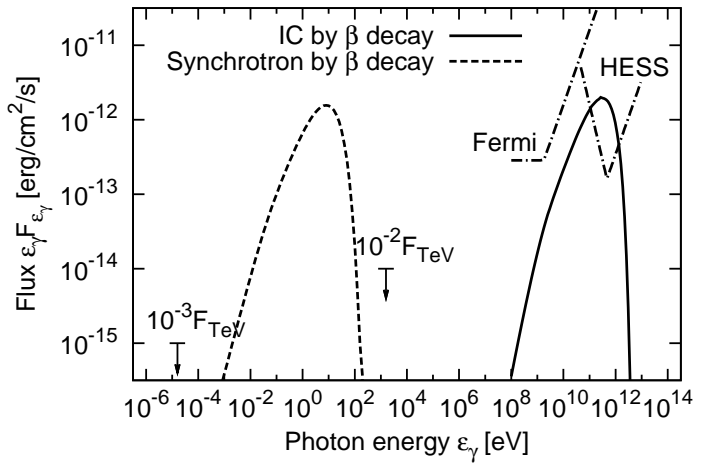

FIG. 2.- Flux from the IC scattering of CMB photons by the $\beta$ decay electrons via the CR neutron component in the GRB jets, compared with the Fermi and HESS sensitivities. We assume a remnant of age $t_{\mathrm{age}}=10^{5} \mathrm{yr}$ at $d=10 \mathrm{kpc}$ with a CR energy $E=3 \times 10^{51} \mathrm{erg}$ [i.e., an old remnant version of Model (I) in Ioka et al. (2004)] and a CR Lorentz factor $10^{6}<\gamma_{n}<10^{9}$ We also show the synchrotron emission from $\beta$ decay electrons for $B=3 \mu \mathrm{G}$, compared with observational upper limits for an $\mathrm{X}$-ray to $\mathrm{TeV}$ flux ratio of $10^{-2}$ and a radio to $\mathrm{TeV}$ flux ratio of $10^{-3}$.

expected total number is $0.1-1$ for a Galactic GRB rate of $\sim 10^{-5}-10^{-6} \mathrm{yr}^{-1}$ (Guetta \& Della Valle 2007). The actual number would be larger because a larger fraction of SNe may have lower-luminosity (LL) jets that were not identified (e.g., Toma et al. 2007), and LL jets can also accelerate CRs to energy $\sim 10^{16-17} \mathrm{eV}$ (e.g., Murase et al. 2006, 2008), which is necessary for the TeV gamma-ray emission. The most optimistic rate consistent with the late-time radio observations is $\sim 10^{-4} \mathrm{yr}^{-1}(\sim 10 \%$ of the SN Ibc rate) (Soderberg et al. 2006a), yielding a total number of $\sim 10$, comparable to that of $\mathrm{TeV}$ unIDs. All of these remnants could be detected, i.e., $d_{\max } \sim 10 \mathrm{kpc}$, if the total energy in accelerated CRs is comparable to the typical GRB energy.

\subsection{Radio-isotope (RI) decay model}

The chemical composition of jets which may be associated with $\mathrm{SNe} /$ hypernovae is currently unknown. One interesting possibility is that they entrain radioactive isotopes (RI), in particular ${ }^{56} \mathrm{Ni}$ and ${ }^{56} \mathrm{Co}$. The SN/hypernova shock leads to explosive nucleosynthesis, predominantly forming ${ }^{56} \mathrm{Ni}$ via complete silicon burning (Maeda and Nomoto 2003). The inner ${ }^{56} \mathrm{Ni}$ would fall back onto the central engine (in those objects where there is one), and a fraction of accreted ${ }^{56} \mathrm{Ni}$ could be ejected with the ensuing jet. The photodisintegration of heavy nuclei would be suppressed in the LL jets whose temperature is less than $\sim \mathrm{MeV}$. Even if heavy nuclei may disintegrate in the innermost accretion disk, ${ }^{56} \mathrm{Ni}$ could be produced again in a cooling wind (MacFadyen 2003). ${ }^{56} \mathrm{Ni}$ could be also entrained into the jet from the surroundings. The subsequent photodisintegration can be avoided in large fraction of parameter space (Wang et al. 2008; Murase et al. 2008). The spectrum and light curve modeling of GRB-associated hypernovae suggests that the total amount of ${ }^{56} \mathrm{Ni}$ is larger than that in ordinary $\mathrm{SNe}$ and heavy elements are aspherically ejected along the jet direction (Maeda and Nomoto 2002).

${ }^{56} \mathrm{Ni}$ decays into ${ }^{56} \mathrm{Co}$ in $t_{\mathrm{Ni}} \ln 2=6.1$ days essentially by electron capture (McLaughlin \& Wijers 2003). The decay proceeds primarily through the excited states of ${ }^{56} \mathrm{Co}$, emitting $\sim 2 \mathrm{MeV}$ gamma-rays. ${ }^{56} \mathrm{Co}$ also decays primarily by electron capture into excited ${ }^{56} \mathrm{Fe}$ with a half-life of $t_{\mathrm{Co}} \ln 2=$ 
77.2 days,

$$
{ }^{56} \mathrm{Co}+e^{-} \rightarrow{ }^{56} \mathrm{Fe}^{*}+\nu_{e},
$$

followed by $\varepsilon_{\mathrm{RI}} \sim 2 \mathrm{MeV}$ gamma-ray emission,

$$
{ }^{56} \mathrm{Fe}^{*} \rightarrow{ }^{56} \mathrm{Fe}+\gamma .
$$

If the nuclei are fully ionized, they decay by emission of a positron $\left(\beta^{+}\right.$decay), e.g.,

$$
{ }^{56} \mathrm{Co} \rightarrow{ }^{56} \mathrm{Fe}^{*}+e^{+}+\nu_{e},
$$

followed by gamma-ray emission, since free electron capture is negligible in our case. The half-life for ionized ${ }^{56} \mathrm{Co}$ is a factor of $\sim 5$ higher than for ${ }^{56} \mathrm{Co}$ with electrons, while that of ionized ${ }^{56} \mathrm{Ni}$ is longer than $3 \times 10^{4} \mathrm{yr}$. Since nuclei can recombine in an expanding jet (McLaughlin \& Wijers 2003), the decay chain ${ }^{56} \mathrm{Ni} \rightarrow{ }^{56} \mathrm{Co} \rightarrow{ }^{56} \mathrm{Fe}$ can start from the ${ }^{56} \mathrm{Ni}$ electron capture.

The outflowing jet is shocked as it interacts with the ISM, leading to particle acceleration. Unlike in the ordinary SNe, the RI acceleration can occur before the RI decay in the objects considered here, involving jets reaching relativistic speeds. Our interest is in the ${ }^{56} \mathrm{Co}$ acceleration, since the accelerated nuclei are completely ionized and the ionized ${ }^{56} \mathrm{Ni}$ is essentially stable (the half-life is longer than $3 \times 10^{4} \mathrm{yr}$ ). For the ${ }^{56} \mathrm{Co}$ acceleration, the shock radius should be smaller than the ${ }^{56} \mathrm{Co}$ decay length, $r<c t_{\mathrm{Co}}(\ln 2) \beta \Gamma$ and larger than the ${ }^{56} \mathrm{Ni}$ one, $r>c t_{\mathrm{Ni}}(\ln 2) \beta \Gamma$, where $\Gamma$ is the Lorentz factor of the jet. Since the shock radius may be estimated by $E_{j} \sim(4 \pi / 3) r^{3} n m_{p} c^{2} \beta^{2} \Gamma^{2}$, we have

$$
2 E_{j, 51}^{1 / 5} n^{-1 / 5}<\beta \Gamma<8 E_{j, 51}^{1 / 5} n^{-1 / 5}
$$

where $E_{j}$ is the total energy of the jet. The Lorentz factor ranges between that of GRBs and hypernovae. Note that the shock radius is relatively large $\gtrsim 10^{16} \mathrm{~cm}$ that no heavy nuclei may disintegrate (Murase et al. 2008; Wang et al. 2008).

Once ${ }^{56} \mathrm{Co}$ is accelerated, the observed mean lifetime is extended by the Lorentz factor,

$$
t_{\gamma}=\gamma t_{\mathrm{iCo}} \sim 10^{5} \mathrm{yr} \gamma_{5}
$$

enabling long-lasting emission, where $t_{\mathrm{iCo}}=5 t_{\mathrm{Co}}$ is the halflife of ionized ${ }^{56} \mathrm{Co}$. The observed energy of the decay gamma-rays is also Lorentz-boosted to

$$
\varepsilon_{\gamma}=\gamma \varepsilon_{\mathrm{RI}} \sim 0.2 \mathrm{TeV} \gamma_{5},
$$

potentially applicable to $\mathrm{TeV}$ unIDs. The ratio of gammaray energy to the ${ }^{56} \mathrm{Co} \mathrm{CR}$ energy is about $f=\varepsilon_{R I} / 56 m_{p} c^{2} \sim$ $4 \times 10^{-5}$. The gamma-ray flux is then estimated as

$$
\varepsilon_{\gamma} F_{\varepsilon_{\gamma}}=\frac{f \zeta E}{4 \pi d^{2} t_{\gamma}} \sim 10^{-12} \mathrm{erg} \mathrm{s}^{-1} \mathrm{~cm}^{-2} \frac{\zeta_{-1} E_{51}}{\gamma_{5} d_{3 \mathrm{kpc}}^{2}},
$$

interestingly comparable to that of TeV unIDs, where $d_{3 \mathrm{kpc}}=$ $d / 3 \mathrm{kpc}$ and $E$ is the total energy of ${ }^{56} \mathrm{Co}$ CRs. In a sense, this is a relativistic version of the $\mathrm{SN}$ emission since the energy source is the RI decay.

In the RI model, a similar way to the $\beta$ decay model, the total source number is $0.1-1$ for a Galactic GRB rate of $\sim 10^{-5}-10^{-6} \mathrm{yr}^{-1}$ (Guetta \& Della Valle 2007), while the most optimistic number is $\sim 10$ for a rate consistent with the late-time radio observations $\sim 10^{-4} \mathrm{yr}^{-1}(\sim 10 \%$ of the
SN Ibc rate) (Soderberg et al. 2006a). We can detect a fraction $\sim(3 \mathrm{kpc} / 10 \mathrm{kpc})^{2} \sim 0.1$ of these sources, if CRs comprise an energy comparable to the typical GRB energy. For a rare event with high energy and/or with reacceleration by the hypernova shock, the observable distance may be farther.

We note that other RI species may also contribute. In particular, the ${ }^{57} \mathrm{Ni}$ emission via $\beta^{+}$decay dominates in the young $\left(\sim 10^{3} \mathrm{yr}\right)$ remnants because its half-life $35.6 \mathrm{hr}$ is $\sim 10^{2}$ times shorter than ${ }^{56} \mathrm{Ni}$ though its yield is less by $0.1-0.01$ (Maeda and Nomoto 2002, 2003).

The shocked ${ }^{56} \mathrm{Co}$ is accelerated to a power-law spectrum $d N_{\mathrm{Co}} \propto \varepsilon_{\mathrm{Co}}^{-p} d \varepsilon_{\mathrm{Co}}$ for $\varepsilon_{\mathrm{Co}}<\varepsilon_{\mathrm{Co} \text {,max }}$. The maximum energy can be $\varepsilon_{\mathrm{Co} \text {, max }} \sim 3 \times 10^{17} \mathrm{eV}$ (e.g., Murase et al.2008), which provides $\varepsilon_{\gamma, \text { max }} \sim f \times\left(3 \times 10^{17} \mathrm{eV}\right) \sim 10 \mathrm{TeV}$ decay gamma-ray. Hereafter we adopt this maximum energy. The Larmor radius of ${ }^{56} \mathrm{Co} \mathrm{CRs}$ is $\sim 4\left(\varepsilon_{\mathrm{Co}} / 10^{17} \mathrm{eV}\right) B_{-6}^{-1} \mathrm{pc}$, leading to the isotropic emission of decay gamma-rays.

As shown in Fig. 3, the decay gamma-rays have a unique spectrum,

$$
\varepsilon_{\gamma} F_{\varepsilon_{\gamma}} \propto \varepsilon_{\gamma}^{-p+1} \exp \left(-\frac{\varepsilon_{p}}{\varepsilon_{\gamma}}\right)
$$

with an exponential cutoff ${ }^{4}$ below

$$
\varepsilon_{p}=\varepsilon_{\mathrm{IR}}\left(\frac{t}{t_{\mathrm{iCo}}}\right) \sim 0.2 \mathrm{TeV}\left(\frac{t}{10^{5} \mathrm{yr}}\right)
$$

and a power-law above it, where the power-law index is softer than that of parent CRs by one. This is because the low energy CRs have already decayed while the high energy CRs are decaying with a rate $(d / d t) \exp \left(-t / \gamma t_{\mathrm{iCo}}\right) \propto \varepsilon_{\gamma}^{-1} \exp \left(-\varepsilon_{p} / \varepsilon_{\gamma}\right)$. The spectrum of $\mathrm{TeV}$ unIDs is characterized as a powerlaw with index 2.1-2.5 (Aharonian et al. 2008), which corresponds to $p=1.1-1.5$ in equation (16). This is slightly harder than the $p=2$ expected in the test particle acceleration case. The harder spectrum could suggest non-linear shock effects in jets where the CR pressure is nonnegligible (Ellison 2001). This is consistent with the fact that the necessary CR energy is comparable to the total energy of jets, $E \sim E_{j}$.

In Figure 3, we also show the synchrotron and IC emission from decay positrons in equation (11), which have an energy comparable to that of decay gamma-rays. The positron spectrum is $d N_{e} \propto \varepsilon_{e}^{-p} d \varepsilon_{e}$ for $\Gamma m_{e} c^{2}<\varepsilon_{e}<\varepsilon_{p}$ and $d N_{e} \propto$ $\varepsilon_{e}^{-p-1} d \varepsilon_{e}$ for $\varepsilon_{p}<\varepsilon_{e}<\varepsilon_{\max }$. The synchrotron frequency is $\nu^{\text {syn }}=q B \varepsilon_{e}^{2} / 2 \pi m_{e}^{3} c^{5} \sim 10^{-4} B_{-6}\left(\varepsilon_{e} / 0.1 \mathrm{TeV}\right)^{2} \mathrm{eV}$ while the IC frequency is $\nu^{\mathrm{IC}} \sim 9 \epsilon_{\mathrm{CMB}}\left(\varepsilon_{e} / m_{e} c^{2}\right)^{2} \sim 10^{8}\left(\varepsilon_{e} / 0.1 \mathrm{TeV}\right)^{2} \mathrm{eV}$ where we consider cosmic microwave background (CMB) as main target photons (Ioka et al. 2004).

However the cooling time $t_{c}=3 m_{e}^{2} c^{3} / 4 \sigma_{T} \varepsilon_{e} U \sim$ $10^{7}\left(\varepsilon_{e} / 0.1 \mathrm{TeV}\right)^{-1} U_{-12}^{-1} \quad \mathrm{yr}$ is usually longer than the decay time $t_{\gamma}$ in equation (13) for $\varepsilon_{e}<\varepsilon_{\max } \sim 10$ $\mathrm{TeV}$ where $U$ is the total energy density of magnetic fields and CMB. Then the luminosity is suppressed by $t_{\gamma} / t_{c} \sim 10^{-2}\left(t_{\gamma} / 10^{5} \mathrm{yr}\right)\left(\varepsilon_{e} / 0.1 \mathrm{TeV}\right) U_{-12}$ compared with decay gamma-rays in equation (15). This is favorable for the interpretation of such sources as $\mathrm{TeV}$ unIDs. Note that the $\sim \mathrm{eV}$ photons by decay positrons come from an extended region in which the optical background dominates the remnant flux.

4 The actual cutoff is a power low $\varepsilon_{\gamma} F_{\varepsilon_{\gamma}} \propto \varepsilon_{\gamma}^{2}$ for $\varepsilon_{\gamma}<\varepsilon_{p}$ because nuclei also make off-axis emission outside the relativistic beaming angle (Ioka \& Nakamura 2001). This does not change our results. 


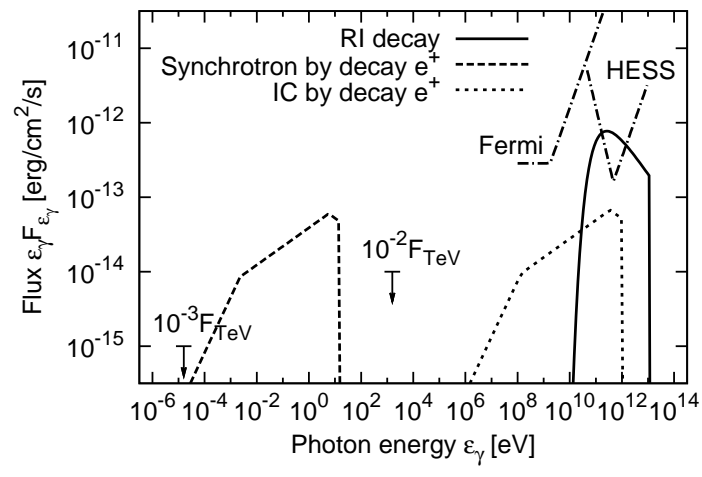

FIG. 3.- Flux of the decay gamma-rays from accelerated RI ${ }^{56} \mathrm{Co}$ CRs in equations (10), 15) and (16), compared with the Fermi and HESS sensitivities. We assume a remnant of age $t_{\text {age }}=10^{5} \mathrm{yr}$ at $d=3 \mathrm{kpc}$ with a CR energy $E=3 \times 10^{51} \mathrm{erg}$ and spectral index $p=1.5$. We also show the synchrotron and IC emission from decay positrons given by equation 11 for $B=3 \mu \mathrm{G}$ and the cosmic microwave background, compared with observational upper limits for an $\mathrm{X}$-ray to $\mathrm{TeV}$ flux ratio of $10^{-2}$ and a radio to $\mathrm{TeV}$ flux ratio of $10^{-3}$.

The accelerated RI CRs may suffer the adiabatic energy losses during the expansion of the remnant (Rachen \& Mészáros 1998), which will greatly suppress the RI emission. This is a potential problem for the RI decay model. However the CR escape from a remnant is not fully understood yet. For instance, it is common to assume the free escape of CRs in models where the GRB reverse shocks are the sites for the ultra high energy CR production (e.g., Waxman 2006; Murase et al. 2008). In the same sense, the RI decay model is a viable possibility for $\mathrm{TeV}$ unIDs.

\section{DISCUSSION}

We have discussed the TeV gamma-ray emission from the $\pi^{0}$ decay, $\beta$ decay and the radio-isotope (RI) decay mechanisms in GRB and/or hypernova remnants, in their possible role as TeV unIDs. There is evidence that typical GRBs predominantly occur in galaxies with less metals than our own (Fruchter et al. 2006; Stanek et al. 2006), although the correlation between the host metallicity and the GRB energy is not confirmed (Savaglio et al. 2008a b). On the other hand broad-lined SNe Ic also inhabit more metal-rich galaxies (Modjaz et al. 2008), suggesting that at least hypernovae and possibly GRB/SNe with LL jets can occur in our Galaxy. In addition, the evidence that GRBs occur in low metal regions could be biased because the GRBs in the metal rich region tend to have no optical afterglows due to the dust absorption (i.e., dark GRBs, which is about half of all GRBs). Then it becomes difficult to identify the hosts for measuring the metal abundance.

To discriminate amongst these models, the imaging of the gamma-ray morphology would be useful. The $\pi^{0}$ decay model predicts a shell structure, since the density inside the remnants is low, while the $\beta$ decay model predicts an elongated structure, and the RI decay model predicts a centerfilled structure. For remnant ages $t_{\text {age }}<10^{5} \mathrm{yr}$, the SNR may be observed also at other wavelengths. HESS J1731-347 may be such an example, which is likely to be an old SNR with a large gamma-ray to radio flux ratio $F_{\mathrm{TeV}} / F_{\text {radio }} \sim 33$ (Tian et al. 2008). HESS J1834-087 may be also an old remnant with an elongated gamma-ray emission outside the SNR (Aharonian et al. 2006), which could be interpreted in the conetxt of a $\beta$-decay model (but see also Mukheriee et al.
(2008))

The $\pi^{0}$ decay model predicts detectable $\mathrm{GeV}$ emission if the spectral index of 2.1-2.4 continues down to the $\mathrm{GeV}$ region (see Fig. 3). Other models predict relatively low levels of GeV emission (Fig. 3, Ioka et al. 2004; Yamazaki et al. 2006; Atoyan et al. 2006), although rare young remnants may be bright if $\varepsilon_{p} \sim 20 \mathrm{GeV}\left(t / 10^{4} \mathrm{yr}\right)$ in the RI model. This may be tested by the Fermi satellite. Note that the GRB prompt and afterglow emission will mask the very early RI decay emission, which becomes prominent later.

The implied TeV neutrino flux ( $\sim$ gamma-ray flux) is not enough for detection by current facilities. However, in the future one may in principle test models through the flavor ratio. We expect $\pi^{+} \rightarrow \mu^{+}+\nu_{\mu} \rightarrow e^{+}+\nu_{e}+\bar{\nu}_{\mu}+\nu_{\mu}$ in the $\pi^{0}$ decay model, no neutrinos in the $\beta$ decay model (since neutrinos are beamed and usually off-axis), and ${ }^{56} \mathrm{Co} \rightarrow{ }^{56} \mathrm{Fe}^{*}+e^{+}+\nu_{e}$ in the RI decay model.

As discussed at the end of $\$ 2.1$, the radio observations limit the $(e / p)_{10 \mathrm{GeV}}$ ratio to $\lesssim 10^{-3}$ in the $\pi^{0}$ decay model. For the RI decay model, the CRs need to freely escape from the acceleration site before suffering the adiabatic energy losses (see the end of $\S 3.2$ ).

The implied CR energy budget is less than $\sim 10 \% \sim$ (10 times energy) $\times\left(10^{-2}\right.$ times rate $)$ of the standard SN CR energy budget in our models. The CRs above the knee at $\sim 3 \times 10^{15} \mathrm{eV}$, however, could be produced mainly by extragalactic and/or Galactic GRBs/hypernovae (Wick et al. 2004; Murase et al. 2006; Wang et al. 2007; Budnik et al. 2008). The chemical composition is increasingly richer in heavy nuclei above the knee $\sim 3 \times 10^{15} \mathrm{eV}$ to the second knee $\sim 6 \times 10^{17} \mathrm{eV}$ (Antoni et al. 2005; Abbasi et al. 2005), and possibly above $\sim 3 \times 10^{19} \mathrm{eV}$ (Unger et al. 2007). These may be accelerated by jets as in the RI decay model.

The GRB remnants may be also responsible for the excesses of cosmic-ray positrons and electrons recently observed by the PAMELA and ATIC/PPB-BETS experiments (Ioka 2008; Fuiita et al.2009). Since the electron and positron sources should be nearby (less than $\sim 1 \mathrm{kpc}$ away), it may be difficult to detect these sources by gamma-rays.

Recently, wide-field optical surveys have been discovering exceptionally luminous SNe, such as SN 2005ap, SN 2008am, SN 2006gy, SN 2006tf and SN 2008es (Miller et al. 2008). The SN 2008es yields a radiated energy of $\gtrsim 10^{51}$ erg and possibly a total energy of $\sim 10^{52} \mathrm{erg}$, comparable to the hypernova energy. The rate could be also comparable to the hypernovae rate although only an upper limit to the rate is obtained (Miller et al. 2008). Therefore such luminous SNe could also become TeV unIDs like the hypernova case, although the luminous SNe are type II and may not be related with GRBs.

In conclusion, we propose that GRB/hypernova remnants are promising candidates for the $\mathrm{TeV}$ unIDs. If $\mathrm{TeV}$ unIDs are hypernova remnants, we can predict more extended (and more numerous) TeV SNRs than observed, which may be discovered by expanding the search region to larger angular scales, even with the current instruments. We also propose a new process of $\mathrm{TeV}$ gamma-ray emission involving the decay of accelerated radioactive isotopes, such as ${ }^{56} \mathrm{Co}$ entrained by relativistic or semi-relativistic jets in GRBs/hypernovae.

We thank T. Nakamura, T. Kamae, S. Razzaque, S. Inoue, R. Mukherjee, C. Dermer, R. Yamazaki, S. Park and T. Mizuno for useful comments. This work is supported 
in part by the Grant-in-Aid from the Ministry of Education, Culture, Sports, Science and Technology (MEXT) of
Japan, No.18740147, 19047004, 21684014 (K.I.), and NASA NNX08AL40G (P.M.).

\section{REFERENCES}

Abbasi, R. U., et al. (HiRes Collaboration) 2005, ApJ, 622, 910

Aharonian, F., et al. 2005, Science, 307, 1938

Aharonian, F., et al. 2006, ApJ, 636, 777

Aharonian, F., et al. 2008, A\&A, 477, 353

Anchordoqui, L. A., et al. 2007, PRL, 98, 121101

Antoni, T., et al. 2005, Astropart. Phys., 24, 1

Atoyan, A., Buckley, J., \& Krawczynski, H. 2006, ApJ, 642, L153

Ayal, S., \& Piran, T. 2001, ApJ, 555, 23

Bamba, A., et al. 2007, Publ. Astron. Soc. Japan 59, S209

Baring, M. G., et al. 1999, ApJ, 513, 311

Budnik, R., Katz, B., MacFadyen, A., \& Waxman, E. 2008, ApJ, 673, 928

Drury, L. O’C., Aharonian, F., \& Völk, H. J. 1994, A\&A, 309, 1002

Ellison, D. C. 2001, Progress of Theoretical Physics Supplement, 143, 125

Fruchter, A. S., et al. 2006, Nature, 441, 463

Fujita, Y., Kohri, K., Yamazaki, R., \& Ioka, K. 2009, arXiv:0903.5298 [astro-ph.HE]

Guetta, D., \& Della Valle, M. 2007, ApJ, 657, L73

Hammer, F., et al. 2006, A\&A, 454, 103

Ioka, K. 2008, arXiv:0812.4851

Ioka, K., \& Nakamura, T. 2001, ApJ, 554, L163

Ioka, K., Kobayashi, S., \& Mészáros, P. 2004, ApJ, 613, L17

Kamae, T., Karlsson, N., Mizuno, T., Abe, T., \& Koi, T. 2006, ApJ, 647, 692

Katz, B., \& Waxman, E. 2008, JCAP, 0801, 018

Kelly, P. L., Kirshner, R. P., \& Pahre, M. 2007, arXiv:0712.0430

Keohane, J. W., Reach, W. T. Rho, J, \& Jarrett, T. H. 2007, ApJ, 654, 938

Konopelko, A., Lucarelli, F., Lampeitl, H., \& Hofmann, W. 2002, J. Phys. G: Nucl. Part. Phys., 28, 2755

Lessard, R. W., Buckley, J. H., Connaughton, V., \& Le Bohec, S. 2001, Astropart. Phys., 15, 1

Liang, E., Zhang, B., Virgili, F., \& Dai, Z. G. 2007, ApJ, 662, 1111

MacFadyen, A. I. 2003, in AIP Conf. Proc. 662, Gamma-Ray Burst and Afterflow Astronomy 2001 (Melville: AIP), 202

Maeda, K., \& Nomoto, K. 2002, ApJ, 565, 405

Maeda, K., \& Nomoto, K. 2003, ApJ, 598, 1163

Matsumoto, H., et al. 2007, Publ. Astron. Soc. Japan 59, S199
McLaughlin, G. C., \& Wijers, R. A. M. J. 2002, ApJ, 580, 1017

Miceli, M., et al. 2006, A\&A, 453, 567

Miceli, M., et al. 2008, Advances in Space Research, 41, 390

Miller, A. A., et al. 2008, arXiv:0808.2193

Modjaz, M., et al. 2008, ApJ, 135, 1136

Mukherjee, R., \& Halpern, J. P. 2005, ApJ, 629, 1017

Mukherjee, R., Gotthelf, E. V., \& Halpern, J. P. 2008, arXiv:0810.1605

Murase, K., Ioka, K., Nagataki, S., \& Nakamura, T. 2006, ApJ, 651, L5

Murase, K., Ioka, K., Nagataki, S., \& Nakamura, T. 2008, Phys. Rev. D, 78, 023005

Naito, T., \& Takahara, F. 1994, J. Phys. G: Part. Phys., 477, 486

Perna, R., Raymond, J., \& Loeb, A. 2000, ApJ, 533, 658

Rachen, J. P., \& Mészáros, P. 1998, Phys. Rev. D, 58, 123005

Reynolds, S. P. 2008, ARA\&A, 46, 89

Savaglio, S., Glazebrook, K., \& Le Borgne, D. 2008a, arXiv:0803.2718

Savaglio, S., Glazebrook, K., \& Le Borgne, D. 2008b, arXiv:0808.2917

Soderberg, A. M., et al. 2006a, ApJ, 638, 930

Soderberg, A. M., et al. 2006b, ApJ, 636, 391

Soderberg, A. M., et al. 2006c, Nature, 442, 1014

Stanek, K. Z., et al. 2006, Acta Astron., 56, 333

Tian, W. W., Leahy, D. A., Haverkorn, M., \& Jiang, B. 2008, ApJ, 679, L85

Toma, K., Ioka, K., Sakamoto, T., \& Nakamura, T. 2007, ApJ, 659, 1420

Uchiyama, Y., Takahashi, T., Aharonian, F. A., \& Mattox, J. R. 2002, ApJ, 571,866

Unger, M., et al. 2007, arXiv:0706.1495

Wang, X. Y., Razzaque, S., Mészáros, P., \& Dai, Z. G. 2007, Phys. Rev. D, 76, 083009

Wang, X. Y., Razzaque, S., \& Mészáros, P. 2008, ApJ, 677, 432

Waxman, E. 2006, Nucl. Phys. Proc. Suppl., 151, 46

Waxman, E., Mészáros, P. \& Campana, S. 2007, ApJ, 667, 351

Wick, S. D., Dermer, C. D., \& Atoyan, A. 2004, Astropart. Phys., 21, 125

Yamazaki, R., Kohri, K., Bamba, A., Yoshida, T., Tsuribe, T., \& Takahara, F. 2006, MNRAS, 371, 1975 